\title{
FAST TRANSIENT ANALYSIS OF OSCILLATORS USING MULTIPLE TIME SCALES WITH ACCURATE INITIAL CONDITIONS
}

\author{
Lei (Lana) Zhu \\ Department of Electrical \\ Engineering, \\ Lakehead University \\ Thunder Bay, Ontario, Canada \\ P $7 B$ 5E1 \\ e-mail: lzhu1@lakeheadu.ca
}

\author{
Carlos E. Christoffersen \\ Department of Electrical \\ Engineering, \\ Lakehead University \\ Thunder Bay, Ontario, Canada \\ P $7 B$ 5E1 \\ e-mail: c.christoffersen@ieee.org
}

\begin{abstract}
A fast and robust method to analyze the transient response of freerunning oscillators is proposed. It is based on the harmonic balance technique derived from the warped multi-time partial differential equation approach. We show for the first time how to precisely obtain initial boundary conditions that are consistent with arbitrary physical initial conditions in the system of ordinary differential equations at any setting time. An adaptive time step technique also reduces the computational effort. The analysis of a LC-tuned bipolar oscillator demonstrates the excellent performance of the proposed method.
\end{abstract}

Keywords - Oscillator Analysis, Initial Condition, WaMPDE, ODE, Multi-Time, Transient Analysis

\section{Introduction}

Transient analysis of oscillators is one important aspect in circuit simulation. The traditional time-domain method using a time-marching approach to solve a system of ordinary differential equations (ODEs) is popular to determine the transient directly. However, in some cases the transient takes a long time to converge to the steady-state, resulting in an extremely expensive computational cost. Several techniques have been proposed to skip the transient and find the steady state directly. We mention here just a few. For example [1] provides a method to find the steadystate of high-Q oscillators using the transient analysis in Spice. In [2], a Newton algorithm is used to find the periodic response and the period of oscillators quickly in lightly damped systems. However, the complete transient solution is still not accessible in these methods. In [3], multiple time axes are employed to separate the fast and slow variations in the response of oscillators, giving a possible way to quickly compute the transient. A variation of that method [4] is known as Warped Multi-time Partial Differential Equation (WaMPDE). The System of ODEs is replaced by a system of partial differential equations. If an accurate transient analysis is required, the initial conditions in the ODEs must be precisely mapped into boundary conditions in the WaMPDE. Usually the boundary conditions in the WaMPDE are approximated using the values of the state variables from one period of ODE or using interpolation to map the ODE results to the WaMPDE domain. However these simple methods to find the boundary conditions are not accurate enough to produce results consistent with the ODE result. This problem has not been treated so far. In this paper we present a method to accurately perform this mapping and thus allow the transient analysis of oscillators with any initial conditions. The key of this work is to build and solve a system of equations where the unknowns are the boundary conditions of the WaMPDE that match the ODE results. Once the correct boundary conditions are found, the WaMPDE method is used to quickly find the transient and steady-state behavior of the oscillator.

The method starts by running a regular time-marching simulation of the oscillator for a short time until some oscillations are produced. The analysis is transformed from ODE to WaMPDE when frequency variation slows down. The ODE solution determines both the initial guess of the local frequency and an approximate mapping between multiple time scales. A new system called boundary condition system is built up considering this mapping and the ODE solution with boundary conditions as unknowns. After solving this system it is possible to obtain the state variables as functions of the two time scales in the subsequent WaMPDE analysis. This procedure transforms a portion of the ODE solution into the WaMPDE boundary condition with accuracy. To reduce the computational cost resulting from large amount of time steps during the WaMPDE simulation an adaptive algorithm is employed to determine the optimum time step size depending on the local truncation error. With accurate boundary conditions and optimum time steps, the transient response is achieved in a precise and quick way.

The proposed method is developed in Section 2. Section 3 presents a case study of a LC-tuned bipolar oscillator. Finally, conclusions are summarized in Section 4.

\section{The Proposed Method}

Assume the oscillator circuit is described by its nodal equations:

$$
G u(t)+C \frac{d u(t)}{d t}+\frac{d Q(u(t))}{d t}+I(u(t))=S(t),
$$

here $u(t)$ is the vector of nodal voltages and selected branch currents, $G$ is a matrix of conductances, $C$ is the matrix representing the linear charge terms, $Q(u(t))$ and $I(u(t))$ 
are vector functions corresponding to the nonlinear devices and $S(t)$ is a vector that represents the sources.

In WaMPDE [4] we must consider at least two time scales: the warped time scale, where the oscillation happens $\left(\tau_{1}\right.$ scale) and another time scale equal to the real time $\left(\tau_{2}\right.$ scale). The relation between $\tau_{1}$ and $\tau_{2}$ is defined by:

$$
\tau_{1}=\int_{0}^{t} \omega_{0}\left(\tau_{2}\right) d \tau_{2}
$$

where $\omega_{0}\left(\tau_{2}\right)$ is the unknown local frequency which is a function of real time $\tau_{2}$. Each element of the $u(t)$ vector is now represented by a function of two time variables $u_{n}\left(\tau_{1}, \tau_{2}\right)$ ( $n$ denotes the node index),

$$
u_{n}\left(\tau_{1}, \tau_{2}\right)=\Re\left\{\sum_{k=0}^{K} U_{n}^{k}\left(\tau_{2}\right) e^{j k \tau_{1}}\right\},
$$

where $k$ is the harmonic number and the period in the warped time scale $\left(\tau_{1}\right)$ is normalized to $2 \pi$ (i.e., $\omega=1$ ). Eq. (1) is now discretized in two time axes:

$$
\left(G+C \Omega_{k}\right) U^{k}+C \frac{\partial U^{k}}{\partial \tau_{2}}+\Omega_{k} Q^{k}+\frac{\partial Q^{k}}{\partial \tau_{2}}+I^{k}-S^{k}=0,
$$

here, $\Omega_{k}$ is a diagonal matrix with nonzero elements equal to $j k \omega_{0}\left(\tau_{2}\right)$. The first two are normally evaluated using the discrete Fourier transform (DFT). Eq. (3) is discretized in the $\tau_{2}$ direction using the Backward Euler (BE) rule or Trapezoidal rule. The resulting algebraic nonlinear system is then solved with the Newton-Raphson method for each value of $\tau_{2}$.

As stated in the introduction, finding accurate boundary conditions is essential in the WaMPDE to obtain the transient analysis. The approach presented in this paper is summarized as follows. Firstly, run the ODE until some oscillations are produced. Secondly, filter one of the state variables from the ODE result to determine an initial guess of the local frequency. A band-pass filter is used to remove the DC component and harmonics higher than the fundamental frequency. A mapping between $\tau_{1}$ and $\tau_{2}$ (like the one shown in Fig. 1) is created according to the zerocrossing points in the filtered waveform since there is only the component of the fundamental frequency left. For example, the first zero-crossing point can be set to represent the phase zero; the second one represents the phase $\pi$; the third one represents the phase $2 \pi$, and so on. We use a straight line to connect these points under the condition of the slowly changing frequency in circuits. This mapping continues until for two or three periods. Linear or polynomial interpolation is used to calculate the state variables at two fixed values of $\tau_{2}(a$ or $b$ ) corresponding to the two vertical lines shown in Fig. 1: $\left(\tau_{1}, a\right)$ and $\left(\tau_{1}, b\right)$. Thirdly, build up the boundary condition equation:

$$
u_{\text {ode }}(t)-\left[u\left(\tau_{1}, a\right)+\frac{u\left(\tau_{1}, b\right)-u\left(\tau_{1}, a\right)}{b-a}(t-a)\right]=0 .
$$

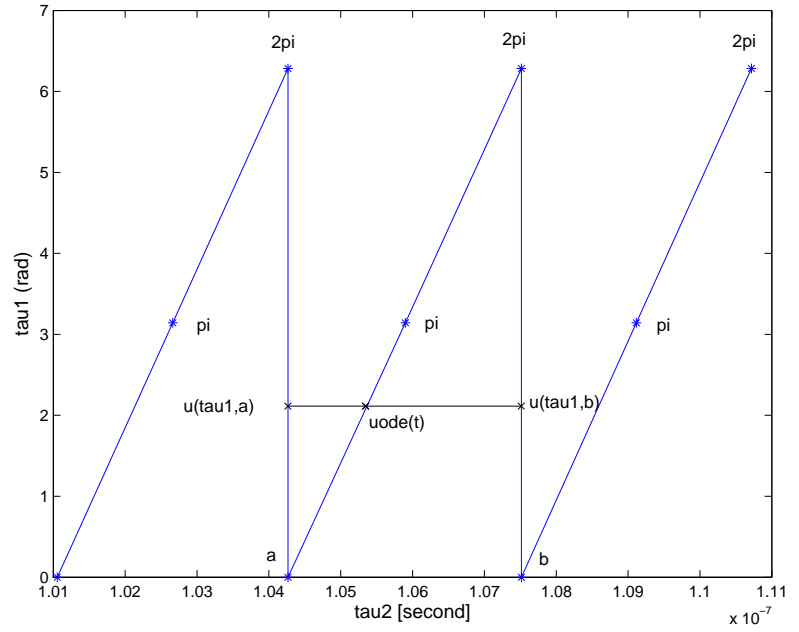

Figure 1: Relation between $\tau_{1}$ and $\tau_{2}$.

Here $t \subset(a, b)$ and $\tau_{2}=t$. After discretization, the vector corresponding to $u\left(\tau_{1}, b\right)$ is calculated using the WaMPDE method with $u\left(\tau_{1}, a\right)$ as boundary conditions. Eq. (3) and Eq. (4) form a system of equations that is solved with the Newton-Raphson method. The unknowns are $u\left(\tau_{1}, a\right)$, $u\left(\tau_{1}, b\right)$ and the local frequency $\omega_{0}(0)$. The local frequency $\omega_{0}(0)$ is an additional unknown that must be calculated for $\tau_{2}=0$. The phase of one of the variables is then fixed to restore the number of unknowns to be equal to the number of equations. That is achieved by setting the imaginary part of one of the variables to be equal to reduce one unknown, such as:

$$
\Im\left(U_{n}^{1}(a)\right)=\Im\left(U_{n}^{1}(b)\right) .
$$

The $b$ value is automatically changed along with the local frequency in each Newton iteration. As a result the time interval of $u_{\text {ode }}$ is adaptively adjusted to improve the accuracy of the final solution.

Fourthly, continue the transient analysis using the WaMPDE method starting with the boundary conditions found before. It is mentioned here that we can restore ODE from WaMPDE at any time. That is easy to implement since the mapping between the two time axes has already been obtained in Eq. (2). As shown in Eq. (3), the periodic solution in the warped time $\tau_{1}$ is solved by the $\mathrm{HB}$ technique. The unknowns are changed to the Fourier coefficients of each node voltage for each value of $\tau_{2}$.

Since the focus is on the transient it is very important to estimate the truncation error of each time step. The local truncation error is calculated by the difference between the result of numerical integration method and the parabolic extrapolation approximation,

$$
\epsilon=\max \left(U_{n}^{k}-U_{n}^{\text {predict }}\right) .
$$

$\epsilon$ is compared with some acceptable value $\epsilon_{\max }$ to evaluate the accuracy of the transient. The result will be rejected if $\epsilon$ is larger than $\epsilon_{\max }$. Adaptive time step control algorithm is used in order to minimize the number of time steps. The 


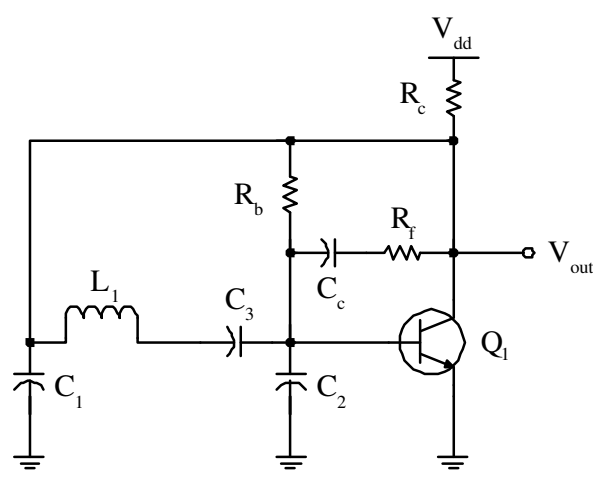

Figure 2: LC-tuned bipolar oscillator.

time-step along $\tau_{2}$ is adaptively changed by the local truncation error which pre-determines the next optimum step size,

$$
h_{n e w}=h_{\text {old }}\left(\frac{\epsilon_{\max }}{\epsilon}\right)^{\frac{1}{m}},
$$

where $m=2$ in linear interpolation and $m=3$ in quadratic interpolation. This results in WaMPDE expression with fewer time steps and therefore substantially reduces the computational cost and improves the accuracy in the transient simulation.

\section{Case Study and Discussion}

The transient analysis of a LC-tuned oscillator employing the proposed method is presented in this section. This example illustrates the main features of the proposed method: precise boundary conditions and faster computational time by using WaMPDE and adaptive time step algorithm. Fig. 2 shows the schematic of a LC-tuned bipolar oscillator, which was one of the autonomous circuit examples in [5]. In this circuit $C_{1}=C_{2}=33 \mathrm{pF}, C_{3}=3.17 \mathrm{pF}$, $C_{c}=560 \mathrm{pF}, L_{1}=100 \mathrm{nH}, R_{f}=680 \Omega, R_{b}=100 \mathrm{k} \Omega$, $R_{c}=1.2 \mathrm{k} \Omega$ and $V_{d d}=10 \mathrm{~V}$.

The amplitude of the output voltage slowly goes up and the transient takes long time compared with the oscillations, part of which is presented in Fig. 3. If we use traditional time-domain method, the expensive computational time is unacceptable. Here we use WaMPDE to solve it. Only a few time steps along $\tau_{2}$ are necessary since the oscillations and the transient variations are decoupled in two different time scales. The boundary condition of each node is solved by the proposed method. In this case, the inductor current was chosen to be the reference variable to decide the WaMPDE beginning time and the initial local frequency. The ODE result of this selected node is filtered in this case by a Butterworth filter for extracting the zerocrossing points. A mapping of multiple times is also established with the zero-crossing points (Fig. 1). Since the local frequency varies very slowly, we assume the straight line between each two of these zero-crossing points.

The transient simulation simply starts from the specified initial conditions and the stop time along $\tau_{2}$ is set to be

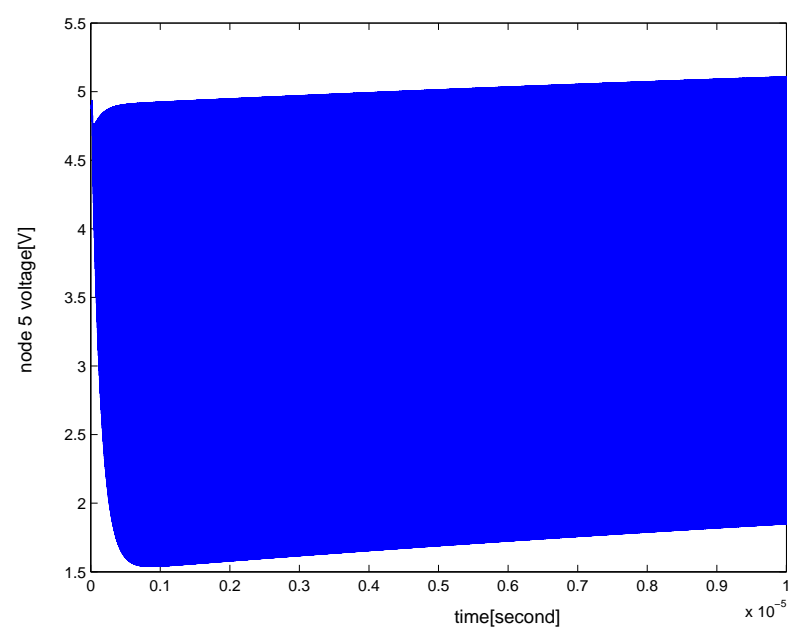

Figure 3: Transient response.

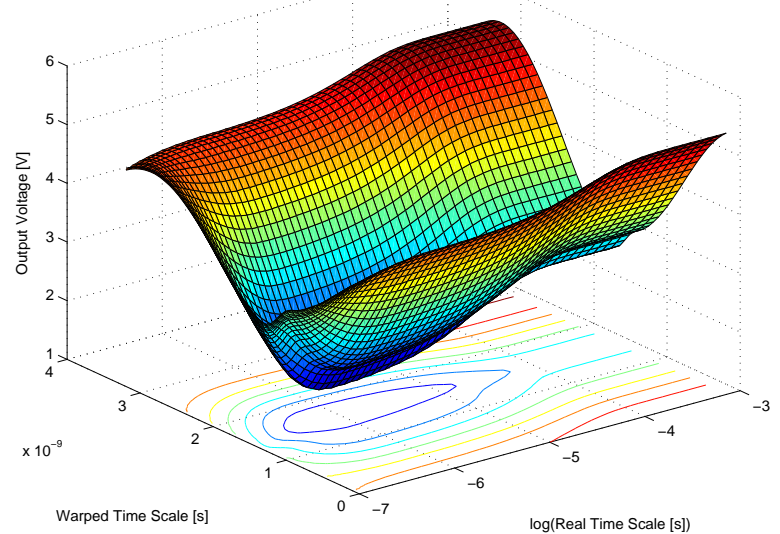

Figure 4: Bi-dimensional representation of output voltage.

$6 \times 10^{-4}$ seconds. The bi-dimensional plot of the output voltage as a function of $\tau_{1}$ and $\tau_{2}$ is shown in Fig. 4. A logarithm scale was used along $\tau_{2}$ to improve the visibility of the samples along that axis. The computational time is orders of magnitude faster than traditional transient analysis. Note that the initial time step is chosen very short but the adaptive algorithm quickly increases its size depending on the local truncation error and thus a only few points are necessary to cover the entire simulation interval. The adaptive time step size is increased along the $\tau_{2}$ time axis and presented in Fig. 5. The oscillator frequency determined by the warped function $\omega_{0}\left(\tau_{2}\right)$ is also represented in Fig. 5. The comparison of the waveforms obtained using the WaMPDE with the proposed boundary conditions and a regular time domain simulation is provided in this study. Very good agreement has been achieved shown in Fig. 6. We also provide the results of WaMPDE simulations using the boundary conditions obtained with the simple approaches discussed in the introduction in Fig. 5 and Fig. 7. The local frequency presents drastic oscilla- 


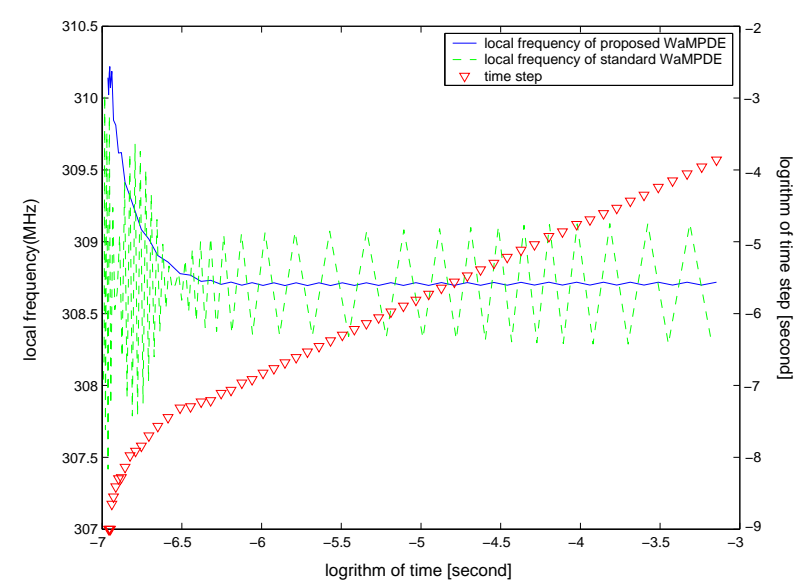

Figure 5. Adaptive time step and the fundamental frequency.

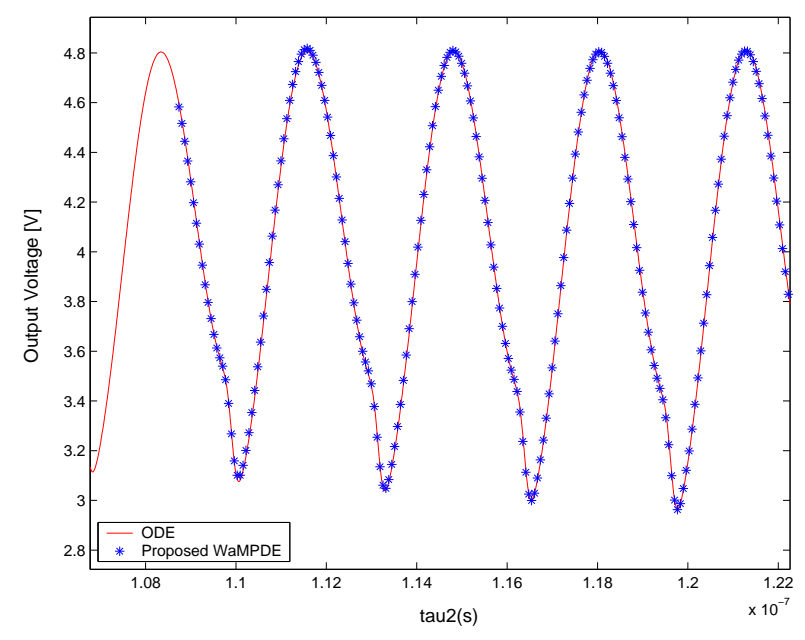

Figure 6. Proposed WaMPDE solution compared to ODE solution.

tions and the agreement of the waveforms is not as good as the proposed WaMPDE, in this case due to a phase shift created by inaccurate boundary conditions. The initial local frequency is $310.141 \mathrm{MHz}$, which is a little larger than the value given by the ODE simulation. After performing many simulations it seems that a spike at the beginning of the WaMPDE simulation is inevitable but it does not influence the final solution since the agreement in all other variables is still very good.

\section{Conclusions}

We have introduced a new method for the analysis of the transient response of oscillators that is fast and accurate. The accuracy of the WaMPDE method depends on the accuracy of the boundary conditions. It was shown for the first time how to obtain precise initial boundary conditions in both the oscillation frequency and the transient solution of each state variable, providing a robust route to switch between multiple time domain and traditional time domain

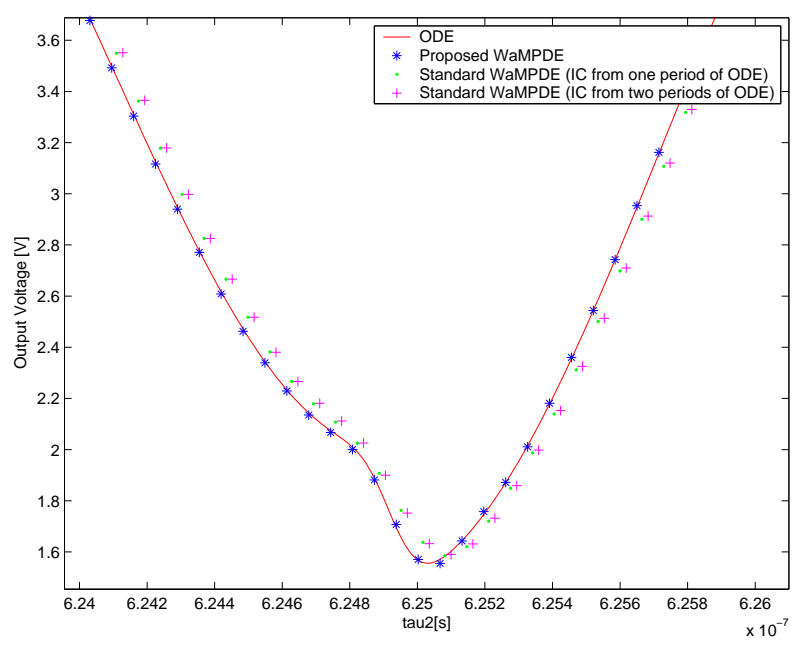

Figure 7: WaMPDE solutions compared to ODE solution.

simulations. An adaptive time step control algorithm is employed and the computational time is orders of magnitude faster than traditional transient analysis.

\section{Acknowledgment}

The authors would like to acknowledge the support of Science and Engineering Research Canada (NSERC).

\section{References}

[1] U. Jorges and G. Jummel, "Finding the steady state of high-Q circuits with proper initial values of the transient analysis," Circuits and Devices Magazine, IEEE, vol.13, Nov. 1997, pp. 6-8.

[2] T. Aprille and T. Trick, "A computer algorithm to determine the steady-state response of nonlinear oscillators," Circuits and Systems, IEEE Transactions on [legacy, pre - 1988], vol.19, Jul 1972, pp. 354-360.

[3] J. Roychowdhury, "Analyzing circuits with widely separated time scales using numerical PDE methods," IEEE Transactions on Circuits and Systems-1: Fundamental Theory and Applications, vol.48, no.5, May 2001, pp. 578-594.

[4] O. Narayan and J. Roychowdhury, "Analyzing oscillators using multitime PDEs," IEEE Transactions on Circuits and Systems-1: Fundamental Theory and Applications, vol.50, no.7, Jul. 2003, pp. 894-903.

[5] A. Brambilla and P. Maffezzoni, "Envelope-following method to compute steady-state solutions of electrical circuits," IEEE Transactions on Circuits and Systems1: Fundamental Theory and Applications, vol.47, Jul 2000, pp. 999-1008. 